\title{
A comparative study of the impact properties of sandwich materials with different cores
}

\author{
K.R. Ramakrishnan ${ }^{1,2}$, K. Shankar ${ }^{1}$, P. Viot ${ }^{2}$, and S. Guerard ${ }^{2}$ \\ ${ }^{1}$ School of Engineering and Information Technology, UNSW@ADFA, Canberra 2600, Australia \\ ${ }^{2}$ Arts et Métiers ParisTech, I2M-DuMAS UMR 5295, 3400 Talence, France
}

\begin{abstract}
Sandwich panels are made of two high strength skins bonded to either side of a light weight core and are used in applications where high stiffness combined with low structural weight is required. The purpose of this paper is to compare the mechanical response of several sandwich panels whose core materials are different. Sandwich panels with glass fibrereinforced polymer face sheets were used, combined with five different cores; polystyrene foam, polypropylene honeycomb, two different density Balsa wood and Cork. All specimens were subjected to low velocity impact and their structural response (Force-displacement curves) were compared to quasistatic response of the panel tested using an hemispherical indenter.
\end{abstract}

\section{Introduction}

Sandwich panels are based on a simple structure of two thin high strength facesheets bonded to either side of a thick low density core such as foams and honeycombs. This provides a lightweight structure with high stiffness. The skins are designed to resist tensile and compressive stresses and are usually made of aluminium or fibre reinforced polymers. The core is designed to resist compression and shear stresses and is usually made of wood, polymer foams, or expanded metal or polymer honeycombs. One of the main drawbacks of these high performance structures is their relatively poor resistance to impact loading [1]. Impact damage in sandwich structures can be caused by tool drops, runway debris, bird strikes, hailstorms or ballistic loading. Ballistic impacts cause localized damage which is clearly visible on inspection while low-velocity impacts involve long contact time between impactor and target which result in global structural deformation with internal damage at points far from the contact region [1]. Bernard and Lagace used Xray photos to determine the extent of delamination damage and noted that extensive delamination and core damage were found in specimens with no visible surface damage [2]. It was recommended by Park et al. [3] that since the damage behaviour of sandwich structures are affected by the mechanical properties of both core and laminated facesheets, the damage behaviour under impact loading deserve careful investigation to ensure the reliability and safety of sandwich structures. Many studies have been performed on low velocity impact of sandwich structures [4-7]. Gustin et al. compared the absorbed energy and maximum impact force of sandwich plates with foamfilled and hollow honeycomb cores subjected to low velocity impact [4]. Cantwell et al. [5] compared the impact response of sandwich structures with balsa wood and PVC foam cores for use in marine applications. Bernard and Lagace studied the impact resistance of composite sandwich plates with graphite/epoxy facesheets and three different cores; aluminum honeycomb, Nomex ${ }^{\circledR}$ honeycomb and Rohacell ${ }^{\circledR}$ foam core using low energy impact tests [2]. Mines et al. studied the perforation due to impact of square sandwich plates with Coremat ${ }^{\circledR}$ and aluminium honeycomb cores [7]. Atas and Sewin [8] compared the low velocity impact response of PVC foam, balsa core sandwich structures with glass fibre reinforced epoxy facesheets. Hazizan and Cantwell [6] compared the low velocity impact response of several polymeric foam core samples with glass fibre/phenolic resin skins and compared the failure modes of the sandwich structures with brittle core and high modulus cores. Castro et al. [9] compared the flexural and impact properties of cork core sandwich composites with Nomex ${ }^{\circledR}$ honeycomb and Rohacell ${ }^{\circledR}$ foam and observed that cork-epoxy agglomerates present a significantly better core shear stress limit, which reduces the crack propagation region.

Mines et al. [7] showed that much of the incident energy of the projectile is absorbed in crushing the core material within a localised region immediate to the point of impact. Bernard and Lagace showed that different cores displayed different damage modes; cell wall buckling in the honeycomb structures while in the Rohacell cores, cracks were formed in the foam parallel to the facesheets [2]. It was suggested by Cantwell et al. [5] that while balsa core had high rigidity and strength, they were prone to fail catastrophically. The authors recommended linear PVC foam for applications involving impact loading because of their ability to absorb energy. Atas et al. observed that polymeric core samples exhibited a higher deformation capability whereas local damage was observed in balsa wood samples instead of delamination [8]. It was suggested by Castro et al. that optimized cork agglomerates have an high energy absorption capacity with minimum damage occurrence and compared favourably with high performance foams in crashworthiness properties [9].

The paper aims to compare the low velocity impact response of sandwich structures, composed of five different core materials; Cork, Polypropylene honeycomb, and polystyrene foam and two different density balsa wood. The facesheets are made of six layers of glass fibre reinforced epoxy. A series of low velocity impact tests are performed using an instrumented drop tower. The force displacement curves of the sandwich panels and the energy absorbed during impact are employed to compare the 
impact resistance of sandwich structures. Quasistatic tests were also performed and the force-displacement curves are compared to those obtained during the impact tests.

\section{Manufacturing of samples}

The sandwich panels with the glass fibre reinforced epoxy skins were manufactured using a wet layup process. The dimensions of the square plates to be tested in the drop tower were fixed as: $150 * 150 \mathrm{~mm}$. 6 layers of plain woven glass fabric with the ply orientation $[0 / 45 / 0]$ s were used. A symmetrical pattern is used in our case in order to have an equal strength in both directions. West System 105Epoxy resin and West System 206- slow hardener were used as the matrix in the supplier recommended ratio of 5 parts resin for 1 part hardener. The fibre/resin weight ratio deduced from the dry weight of the fabric and the final weight of the cured samples, was approximately $60: 40$. The two facesheet layers of glass fibre were bonded on each side of the core material and the sandwich panel was co-cured at room temperature under a vacuum bag for 24 hours. Co-curing the facesheets ensured good adhesion with the core. The nominal thickness of the cured sandwich panels were $14 \mathrm{~mm}$, with a $10 \mathrm{~mm}$ thick core and $2 \mathrm{~mm}$ thick facesheets. To facilitate the manufacturing of several samples required for the experiments, the core material was cut to $450 * 450 \mathrm{~mm}$, bonded to the facesheets, and the cured panel was cut into 9 square plates of $150 \mathrm{~mm}$ by a diamond saw.

\section{Experimental setup}

The sandwich panels with the 5 different cores were tested under two conditions; quasistatic bending or indentation and low velocity impact. The quasistatic response of the sandwich composite panels to indentation with hemispherical impactor was studied in order to compare the response with that of low velocity impact. Sandwich panels with glass fibre-reinforced polymer (GFRP) face sheets and the five different cores were quasistatically loaded at a constant displacement rate of $5 \mathrm{~mm} / \mathrm{min}$ in a electro-mechanical machine (Zwick Roell, Germany).

Low velocity impact testing was accomplished using a drop tower. The drop tower setup consists of an instrumented impactor that is secured to a carriage that falls along guideposts and collides with the plate. An electromagnet holds the carriage lifted to a pre-determined height and the impact event is initiated by switching off the electromagnet and letting the carriage fall freely under the action of gravity. The impactor head transfers the impact energy to the test specimen. The drop height is varied to give a range of impact energies while the mass of the impactor is kept constant. The maximum impact energy that can be obtained is limited by the weight of the impactor carriage and the maximum height of the tower. The drop tower setup used for the low velocity impact testing is shown in Fig. 1.

The transducers used in the drop tower are a force sensor, an accelerometer and velocity counters. The dynamic force sensor and the accelerometer provide force-time and acceleration-time history of the impact. Initial velocity

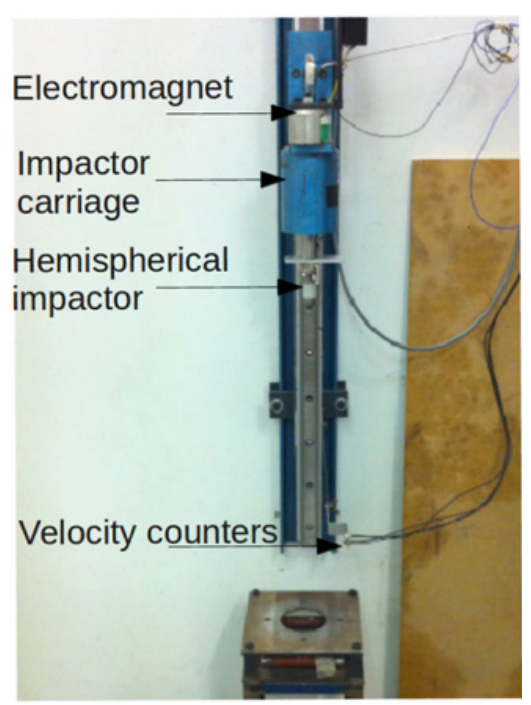

Fig. 1. Impactor carriage in the drop tower setup.

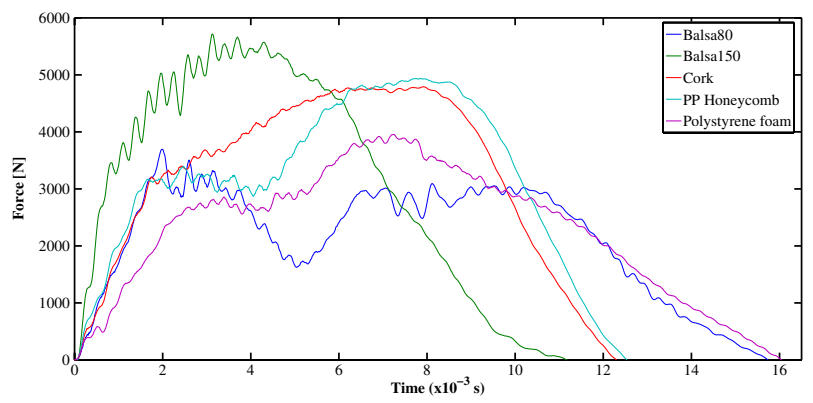

Fig. 2. Typical force-time history of low velocity impact on sandwich panels $(50 \mathrm{~J})$.

is found from data from the velocity counters, which can be used to calculate kinetic energy of the impactor and also provide initial values for the velocity-time and displacement-time histories. Data from the sensors were acquired at the rate of 100000 samples per second per channel for 0.1 second giving us a total of 10000 data points. The acceleration data (deceleration in this case) can be converted to force by multiplying it by the total mass of the impactor carriage assembly. A pair of infrared emitting LED and phototransistor supplied by Omron ${ }^{\circledR}$, Kyoto, Japan was used as velocity counters. The velocity immediately before the impact event was deduced from the time taken for the projectile to travel between the two LEDs separated by a known distance of $12 \mathrm{~mm}$.

The $20 \mathrm{~mm}$ diameter hemispherical headed impactor strikes the centre of the square target plate which is clamped with solid steel annular plates with internal diameter of $135 \mathrm{~mm}$. The bolts were tightened to a torque of $5 \mathrm{Nm}$, in order to provide consistent clamping from experiment to experiment. The initial energy of the impact was calculated from the mass of the impactor $(8 \mathrm{~kg})$ and initial impact velocity found from the velocity counters. The sandwich panels were tested for three velocities, 2.5 , 3.5 and $4.3 \mathrm{~m} / \mathrm{s}$ corresponding to impact energies; $25 \mathrm{~J}, 50 \mathrm{~J}$ and $75 \mathrm{~J}$. Typical force-time history of low velocity impact is shown in Figure 2, corresponding to the 50J impact. It can be seen that the load versus time curves of all sandwich composites show the same tendency, namely, the force 


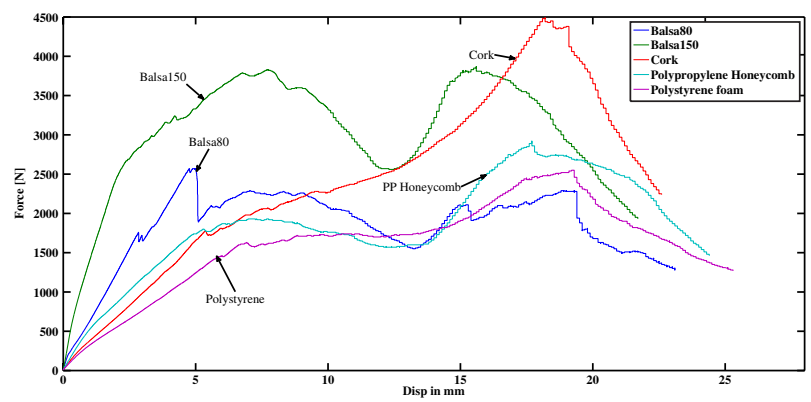

Fig. 3. Quasistatic Force-displacement curve of sandwich samples.
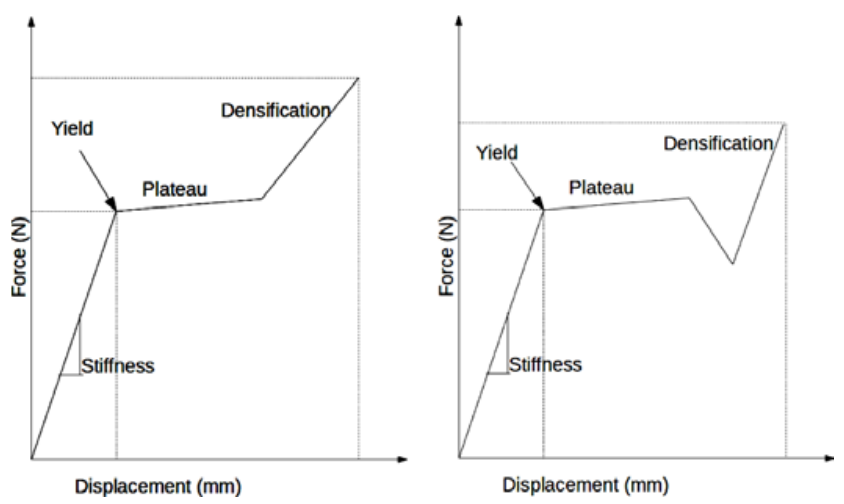

Fig. 4. Idealised behaviour of the loading part of the Forcedisplacement curve of sandwich samples.

history curve exhibiting a linear increase as the indenter contacts the specimen and is followed by a prolonged contact with the core. There is a sudden drop in the load after it reached the first peak value for sandwich plate with Balsa80 core, which indicates a loss in stiffness due to delamination or brittle cracks in the core.

\section{Results}

\subsection{Quasistatic bending}

It is observed that the load-indentation curve of the sandwich panels in Figure 3 showed a quasi-linear behaviour for low values of indentation, followed by a non-linear regime. It is also clear from the force- displacement curve that the stiffness of the sandwich panel, i.e. slope of the linear portion of the curve, is different for the plates with different cores, implying that the behaviour of the sandwich plate is dependant on the core properties even for low values of displacement. The behaviour of the sandwich after yield exhibits a core-dominant behaviour and follows regions of plastic plateau and densification of the core. The loading behaviour of the sandwich plate can be idealised as shown in Figure 4. It can be seen that while sandwich plates with cork, polypropylene honeycomb and polystyrene foam exhibit only three regions - linear part, plateau and densification, the sandwich plates with balsa wood core show a drop in force that perhaps corresponds to the delamination of the facesheets or the crack propagation in the core. The delamination between the facesheet and

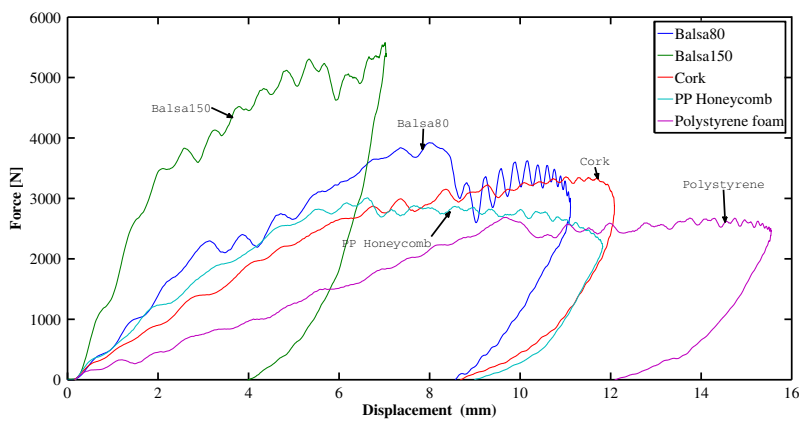

Fig. 5. Force-displacement curve for $25 \mathrm{~J}$ impact.

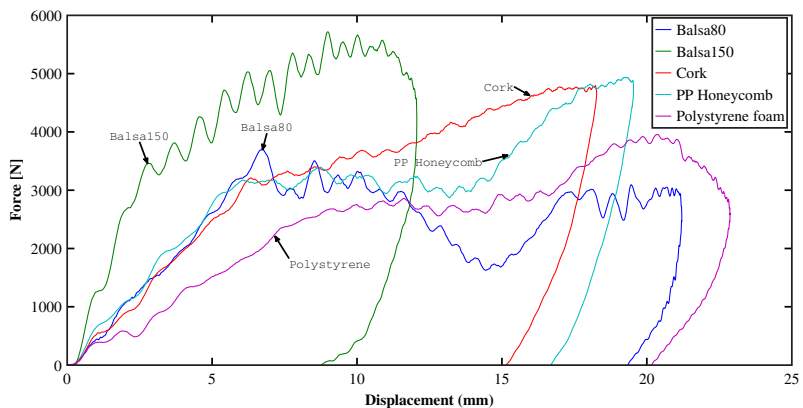

Fig. 6. Force-displacement curve for $50 \mathrm{~J}$ impact.

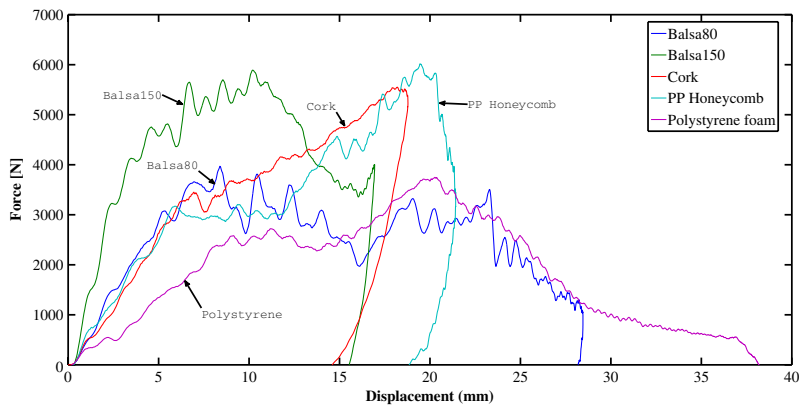

Fig. 7. Force-displacement curve for $75 \mathrm{~J}$ impact.

the core that is observed in the balsa core sandwich plates is not observed in the other sandwich materials.

It can be surmised from the quasistatic test that the stiffness, yield point and rupture point are of particular interest for comparison of the different cores.

\subsection{Low velocity impact}

Figure 5 shows the force- displacement plot for the different sandwich composites at $25 \mathrm{~J}$ impact energy. And Figures 6 and 7 show the force- displacement plots for the sandwich plates at 50 and $75 \mathrm{~J}$ impact energy, respectively. It can also be seen that the force-displacement curves obtained from impact have similar trend to the curves from the quasistatic indentation tests. The behaviour of the sandwich is however different under impact, including the extent of damage and quantitaive values of stiffness, yield force, etc. It can be seen that the Balsa150 core sandwich has consistently the highest stiffness and the polystyrene foam the lowest. The prolonged plateau region indicates the interaction of the indenter through the foam 


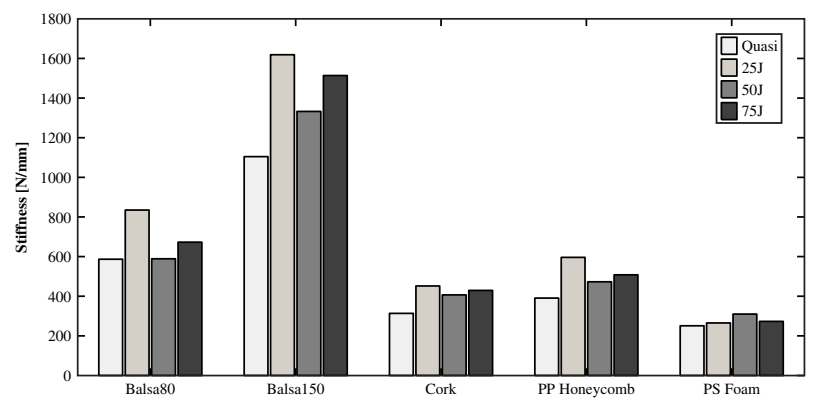

Fig. 8. Stiffness of the initial linear part of Force-displacement curve.

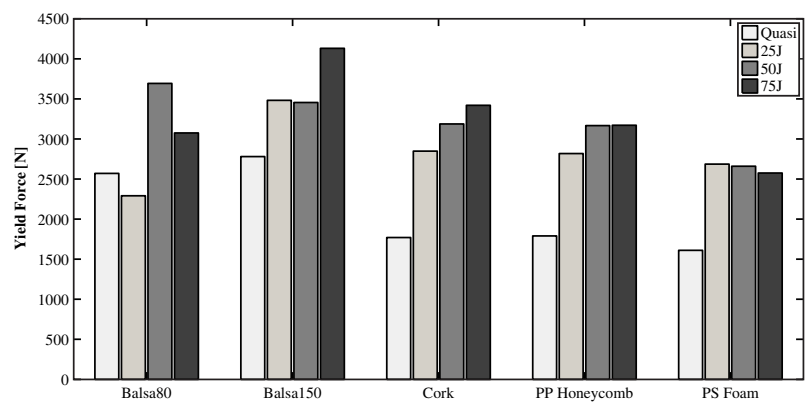

Fig. 9. Yield force of the sandwich samples.

core. For the samples where the indenter penetrates the core and makes contact with the bottom face sheets, a secondary peak value is observed, representing the load carried by the bottom face sheets; then the specimens are completely penetrated, leading to structural failure. Core damage in the foam consisted of core crushing and cracks in the foam while the damage in the honeycomb consisted of the buckling of cell walls. According to Park et al. [3], the impact damage in sandwich structures is mainly delamination of peanut-shaped with major axis along lower fiber orientation. It was observed that an extensive foam core crushing in the area under the indenter produced a rapid decrease in the sandwich panel stiffness which is consistent with the results presented by Rizov et al. [10]. The stiffness, yield force and energy, peak force, maximum displacement and energy absorbed are considered for comparison.

\section{Discussion}

Figures 8 and 9 shows the stiffness of the initial linear portion and the yield force at which the plateau begins. It can be seen that the stiffness of the force-displacement curve is not constant across different loading conditions. There is a variability which is more pronounced in the two balsa samples than in the other cores. Polystyrene has a lower stiffness than other sandwich plates and has a lowest yield force among the sandwich samples for 50 and $75 \mathrm{~J}$ impact loads. However, the sandwich plate with polystyrene foam has high value of displacement before yielding, which increases the energy required to produce yielding in the sandwich, shown in Figure 10. It is seen that sandwich with Balsa150 core, despite having the highest stiffness, requires less energy to yield than sandwich with polystyrene foam core.

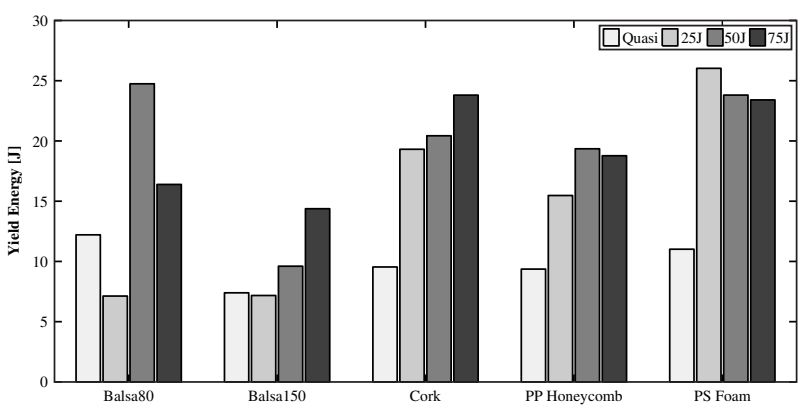

Fig. 10. Energy required to initiate yield of the sandwich samples.

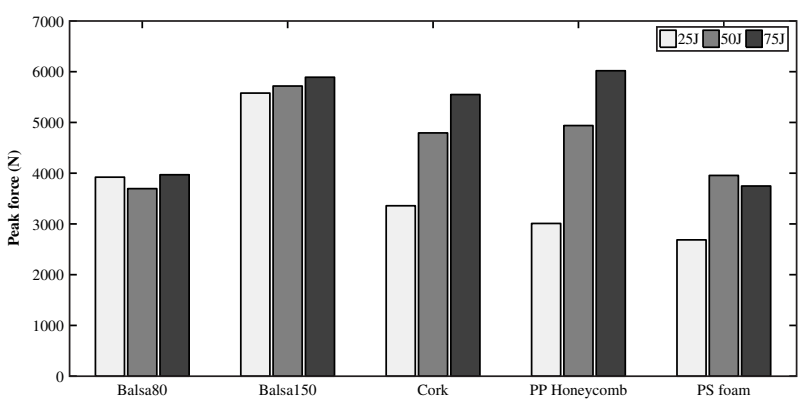

Fig. 11. Peak force of the sandwich samples subjected to low velocity impact.

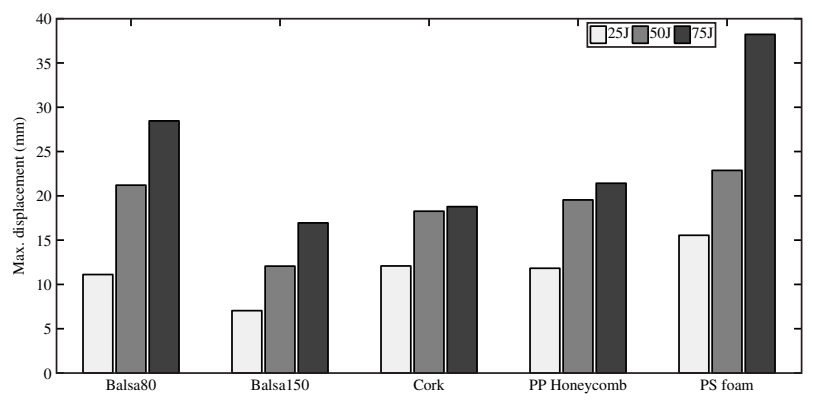

Fig. 12. Maximum displacement of the sandwich samples subjected to low velocity impact.

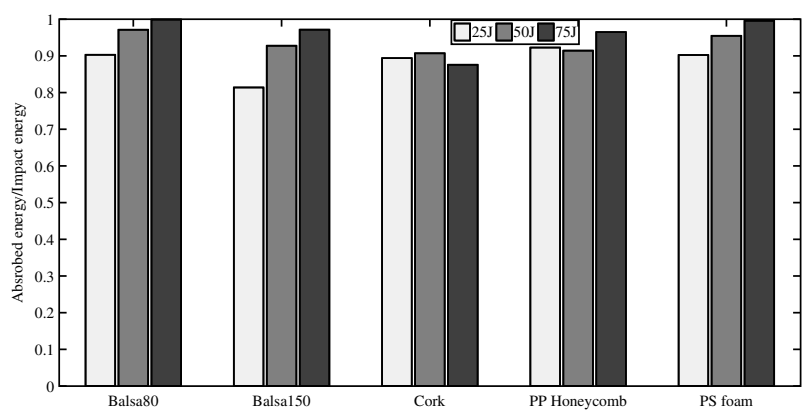

Fig. 13. Absorbed Energy/Impact Energy of different sandwich samples.

Figure 12 shows the maximum displacement which shows an increasing trend for all the sandwich samples. Similarly, the peak force value shown in Figure 11 exhibits an increasing trend with higher loads except for the balsa samples which have almost constant peak force.

The ratio of energy dissipated and initial impact energy shown in Figure 13 provides a normalised value with which to compare the different core sandwich composites. 


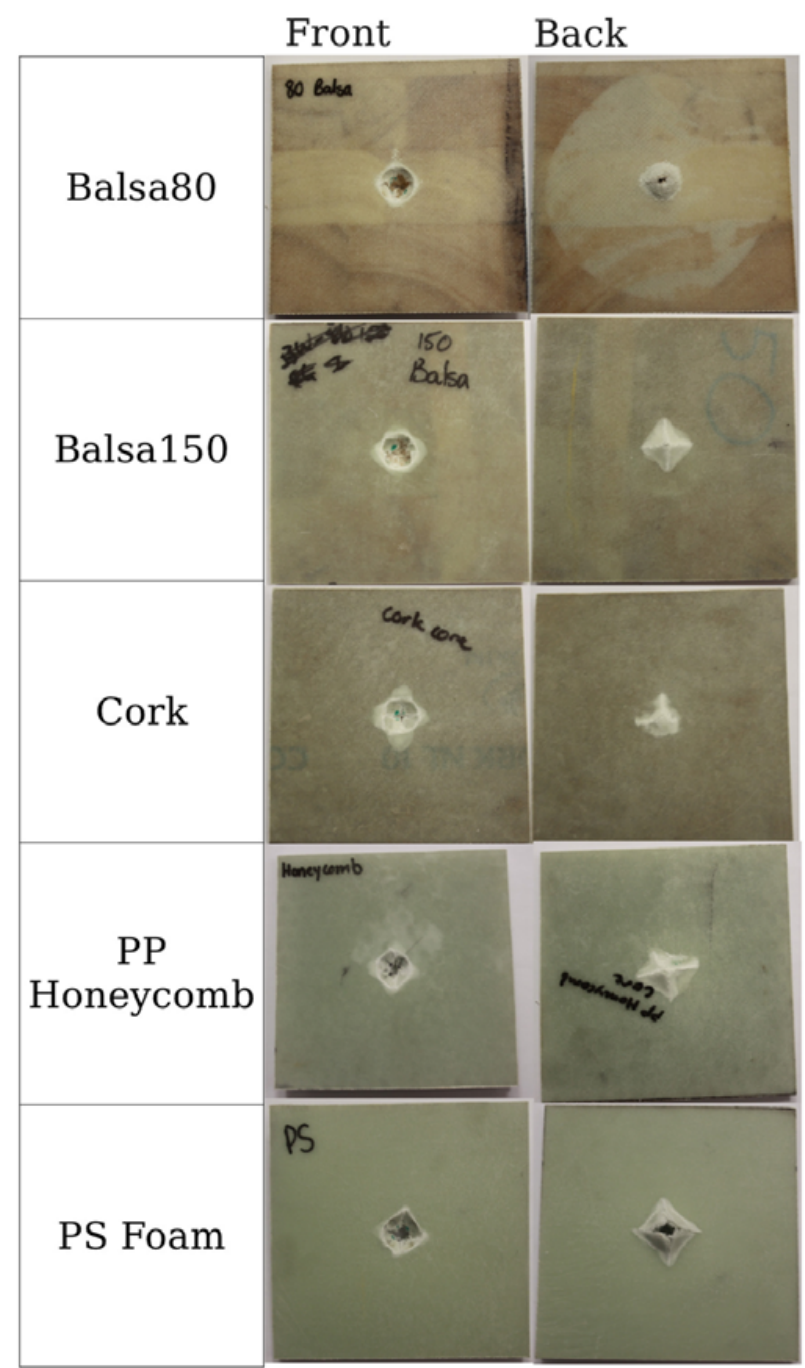

Fig. 14. Impacted samples of different sandwich materials.

The value of this ratio varies from 0 to 1 , where 1 implies that all the kinetic energy of the impactor is absorbed by the sandwich plate in various damage mechanisms. It is hypothesised that the less energy absorbed by the sandwich plate during the impact, the more energy is returned to the impactor in its rebound. This would lead to the conclusion that the sandwich composite with the least value of this energy ratio is most suited for applications requiring impact resistance. It is seen that Cork core sandwich has the lowest value for all the three loading energies.

A subsequent visual examination of the impacted samples highlight three prominent modes of failure. Energy absorption is maximized through the sandwich construction, as penetration through all the three components (top facesheet, core, and bottom facesheet) is required for the complete failure of the sandwich structure. It was observed that for many cases with considerable damage in the top facesheet, the lower facing of the sandwich structure was left undamaged. It was also observed that there was no damage in the bottom facesheet unless there was penetration of the top facesheet and extensive core crushing or if the tensile stresses were high. Initial damage in the low density foam sandwich structures took the form of shear cracking in the brittle foam core. Failure in the intermediate modulus systems occurred as a result of core buckling close to the point of impact. The peanut shaped damage with its centre at the point of impact and axes along the fibre direction is observed. Figure 14 shows the front and rear surface of the impacted samples for the $75 \mathrm{~J}$ impact. Finally, failure in sandwich structures with a higher modulus cores took the form of delamination in the top surface skin. The sandwich samples with Balsa80 wood core and to a lesser extend the Balsa150 core sandwich, show a clear area of delamination, represented by a lighter colour in the picture. All other sandwich panels exhibit a very localised damage around the area of the impactor. It can be seen that for the $75 \mathrm{~J}$ impact there is considerable rear skin damage for all sandwiches except for the sandwich with cork core. This is consistent with the energy absorbed curve shown in Fig. 13. Cork core sandwich plate has absorbed the least amount of energy and shows the least amount of damage. It should however be noted that the densities of the various cores vary from $32 \mathrm{~kg} / \mathrm{m}^{3}$ for the polystyrene foam to $150 \mathrm{~kg} / \mathrm{m}^{3}$ for the Balsa150 core and the sandwich panels tested in the present study were of the same thickness and not normalised for weight. Therefore, in applications where the weight is of primary importance, a low density foam like polystyrene may be more appropriate. In addition to being lighter, polystyrene is capable of absorbing a lot of energy before yielding. A high density balsa core may also be more suitable in applications where stiffness is the most important criteria.

\section{Conclusion}

This paper looked at the impact behaviour of different configurations of sandwich structures subjected to low velocity impact loading. A quasistatic indentation test was done to observe the various phases of the loading curve. The impact testing was done using an instrumented drop tower. The data obtained from the tests were used to compare how the panels performed in relation to each other. The stiffness of the glass fibre facesheets allows the samples to resist higher impact levels without large deformation but beyond a certain input energy, the damage to the sample is irreversible. A comparison of sandwich panels in terms of the energy absorbed by the panel was undertaken. The aim was to determine the type of sandwich panel that performed best under impact loading and cork core was considered the most promising core material based on the amount of energy absorbed. The sandwich plates with cork core absorbed the least amount of energy and also exhibited less damage than the other sandwich samples for higher load condition. However, it was discussed that depending on the type of application, a lower density foam core may also be suitable.

\section{References}

1. M. Bhuiyan, M. Hosur, S. Jeelani, Composites Part B: Engineering 40(6), 561 (2009) 
2. M.L. Bernard, P.A. Lagace, Journal of Reinforced Plastics and Composites 8(5), 432 (1989)

3. J. Park, S. Ha, K. Kang, C. Kim, H. Kim, Journal of Materials Processing Technology 201(13), 425 (2008)

4. J. Gustin, M. Mahinfalah, G.N. Jazar, M. Aagaah, Composites Science and Technology 44(6), 574 (2004)

5. W. Cantwell, C. Dirat, P. Davies, SAMPE Journal 30(4), 45 (1994)
6. M.A. Hazizan, W.J. Cantwell, Composites Part B: Engineering 33(3), 193 (2002)

7. R. Mines, C. Worrall, A. Gibson, International Journal of Impact Engineering 21(10), 855 (1998)

8. C. Atas, C. Sevim, Composite Structures 93(1), 40 (2010)

9. O. Castro, J. Silva, T. Devezas, A. Silva, L. Gil, Materials and Design 31(1), 425 (2010)

10. V. Rizov, A. Shipsha, D. Zenkert, Composite Structures 69(1), 95 (2005) 